SESSION III: IMMUNODIAGNOSIS

CHAIRPERSON: PROFESSOR PATRICK BRENNAN 


\title{
South India immunoprophylaxis trial against leprosy: relevance of the findings in the context of trends in leprosy
}

\author{
M. D. GUPTE \\ National Institute of Epidemiology, Chetput, Chennai, India
}

\section{The South India vaccine trial}

Our group conducted an immunoprophylaxis trial in South India among 171,400 volunteers. ${ }^{1}$ The trial, launched during 1991, compared four vaccines: (i) a combination of BCG and heatkilled Mycobacterium leprae (HKML), (ii) the ICRC bacillus, (iii) Mycobacterium $w(\mathrm{Mw})$, and (iv) BCG, and employed normal saline as a placebo. As shown in Figure 1, the protective efficacy of the vaccines, judged on the basis of the results of the second resurvey, was: $\mathrm{BCG}+\mathrm{HKML}, 64 \%$ [95\% confidence interval (CI) 50.4-73.9]; the ICRC vaccine, $65 \cdot 5 \%$ protection (CI $48 \cdot 0-77 \cdot 0$ ); $\mathrm{Mw}, 25 \cdot 7 \%$ protection (CI $1 \cdot 9-43 \cdot 8$ ); and $\mathrm{BCG}, 34 \cdot 1 \%$ protection (CI 13.5-49.8).

In view of the extensive use of BCG in tuberculosis control and the results of several trials of BCG against leprosy, BCG was used more as a control than as an active vaccine. The efficacy of BCG observed in the present trial was consistent with the results of an earlier trial in an adjacent area, in which BCG was found to confer $24 \cdot 4$ (CI 20.9-27.8)\% protection. $^{2}$

By comparison with the incidence of leprosy noted among those administered placebo, in the first survey, conducted immediately following vaccination, all of the vaccines, including BCG, showed a 'negative' effect, which was not statistically significant. The negative effect, shown in Figure 2, of BCG + HKML was $7.6 \%$ that of ICRC 6.9\%, that of Mw $11.5 \%$, and that of BCG $28.7 \%$. The negative effect had been observed earlier in several other studies, including the south India trial of BCG against tuberculosis and the Burma trial. ${ }^{3,4}$ If the trial design had not included a group administered placebo, it would have been impossible to demonstrate the negative effect of the vaccine preparations, as was the case in the Venezuela and Malawi trials. ${ }^{5,6}$

It is difficult to explain the negative effect. Fine has suggested that this negative effect may result from accelerated progression to mycobacterial disease among those already infected at the time of vaccination, ${ }^{7}$ and some workers have suggested that precipitation of disease by vaccination could be considered an indication of efficacy of the vaccine. In our vaccine trial, the two vaccine preparations that demonstrated the smallest negative effect in the first resurvey yielded the greatest protective effect in the second resurvey. Whatever the explanation of the negative effect, it may not be appropriate to combine the results of the two resurveys to provide an estimate of the efficacy of each of the vaccines. 


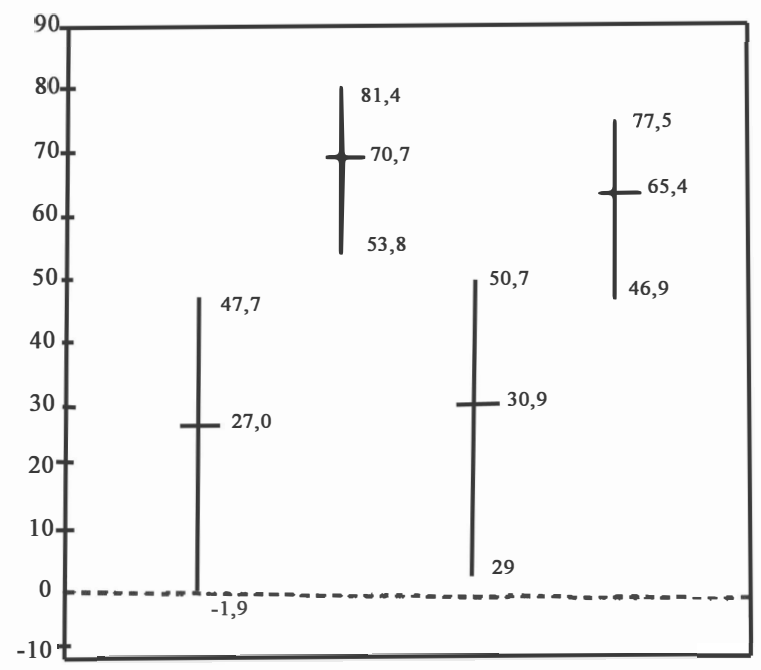

Figure 1. Results of the second resurvey. The values on the ordinate are those of percent protective efficacy. First bar represents BCG, second bar, BCG + HKML, third bar, Mw and fourth bar, ICRC.

We are presently carrying out the third resurvey. Should we find efficacy of the same levels as those observed in the second resurvey, one could certainly make a strong case for use of the effective vaccine in the prevention of leprosy.

There were several other interesting features in this study. Prior vaccination with BCG, indicated by the presence of BCG scars, did not appear to influence substantially the efficacy of the vaccines. In addition, the efficacy of the several vaccines was not influenced by age. It appears that, in a population highly endemic for leprosy, such as that in the south India

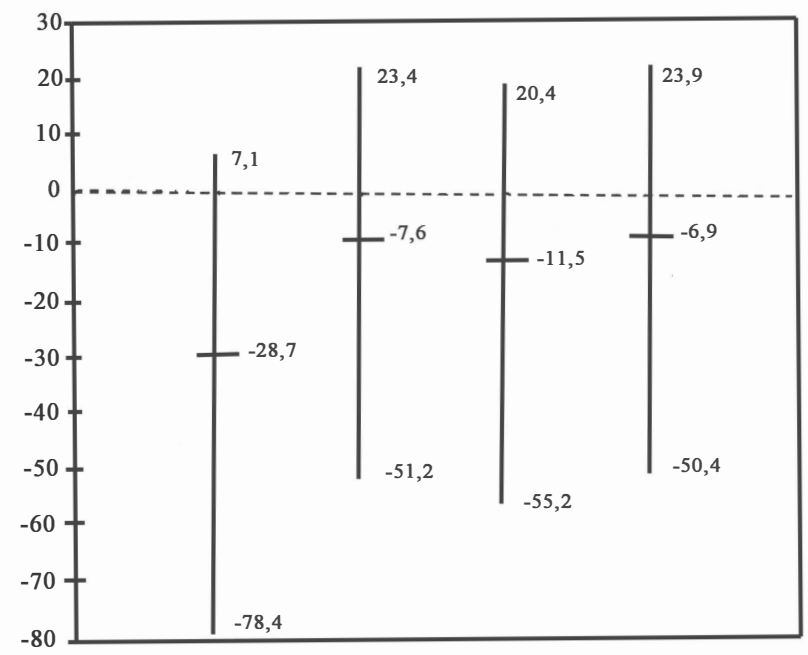

Figure 2. Results of the first resurvey. The values on the ordinate are those of percent protective efficacy. First bar represents BCG, second bar, BCG + HKML, third bar, Mw and fourth bar, ICRC. 
vaccine trial area, the efficacy of the vaccines was not related to prior infection by either M. leprae or some environmental mycobacterium. Finally, that both ICRC and BCG + HKML, which are killed vaccines, produced significant levels of protection is inconsistent with the widely held belief that only live vaccines are protective. ${ }^{7}$

At the time this trial was launched, the expected incidence of leprosy was greater than that actually observed. Because the incidence of multibacillary forms of leprosy was rather low, it was not possible to study the prophylactic efficacy of any of the vaccine preparations against these more serious forms of the disease.

A prophylactic efficacy of $65-70 \%$ may certainly be considered a promising result from the public health point of view. The future use of BCG + HKML appears unlikely, because armadillo-derived $M$. le prae are not likely to be available in quantities sufficient for vaccine preparation. On the other hand, the ICRC vaccine appears to offer considerable potential as a prophylactic agent for the future. At present, the taxonomy of the ICRC bacillus is not very clear. It is necessary to undertake studies to characterize the ICRC vaccine at the earliest opportunity.

The possibility of a second-generation vaccine against leprosy has been suggested. However, to conduct vaccine trials in leprosy, populations with high levels of endemicity and incidence are required. Moreover, vaccine trials are very time-consuming and financially demanding. Finally, because there exist at least two good vaccines that meet public health needs, it will not be possible to conduct placebo-controlled vaccine trials. For these reasons, the prospects for conducting new trials of leprosy prophylaxis are bleak. However, there could be opportunities to make observations for leprosy as well in a trial of a secondgeneration tuberculosis vaccine.

\section{Is there a need for a vaccine against leprosy?}

The prevalence of leprosy has declined considerably since the worldwide implementation of the strategy of multidrug therapy (MDT). In September 1999, the global prevalence of leprosy was approximately 1.4 per 10,000 population. ${ }^{8}$ However, new-case detection rates have not declined perceptibly at the global level. In a Workshop conducted by the Indian Association of Leprologists in Chennai in 1993, several data sets from the country were examined. As shown in Figure 3, very interesting patterns of recorded prevalence and new-case detection rates emerged from these studies. ${ }^{9}$ The analysis demonstrated that, despite a rapid decline of prevalence following implementation of MDT, new-case detection rates remained quite stable. In addition, our study of the trends of new-case detection in several countries demonstrated clearly that MDT did not produce a precipitous decline. ${ }^{10}$ There is, however, a steady and almost imperceptible declining trend of new-case detection. Because, as a result of single-dose therapy, single-lesion cases are not included in the prevalence of leprosy, and because the fixed-duration MDT regimens are short, prevalence rates may be expected to remain at low and constant levels. It may be possible to strengthen leprosy control programmes in an integrated manner, in order to clear still-existing 'backlogs' of leprosy patients. However, the presently observed trends do not suggest that leprosy will be eliminated or eradicated in the near future. Therefore, one can make a strong case for the use of anti-leprosy vaccines to control or eradicate leprosy.

In collaboration with Erasmus University in Rotterdam, The Netherlands, we have developed a simulation model for leprosy. ${ }^{11}$ Our recent work with this model has involved 


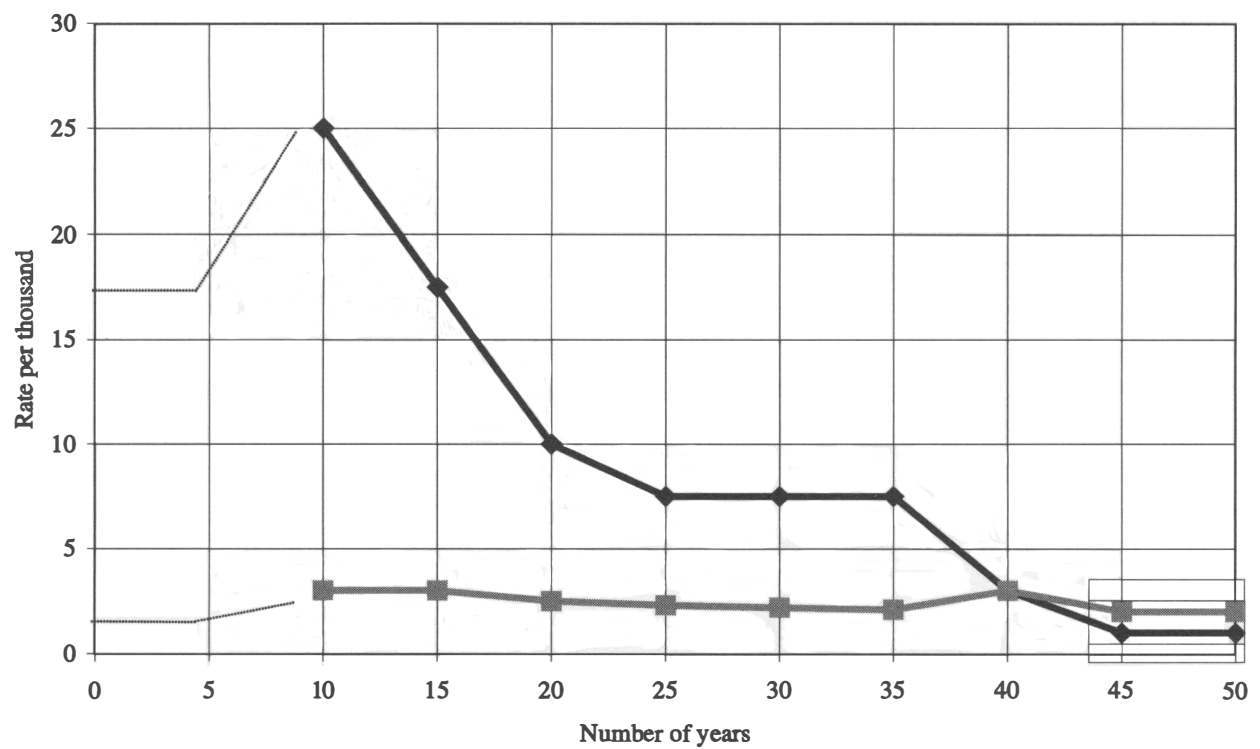

Figure 3. Effect of dapsone and MDT interventions on the prevalence and incidence of leprosy. $\downarrow---$ : recorded

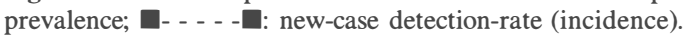

intervention with a prophylactic vaccine that confers $65 \%$ protection. ${ }^{12}$ As shown in Figure 4, introduction of a vaccine could bring about a dramatic decline of leprosy incidence, and, at least in the context of this model, make it possible to conceive of the eradication of leprosy.

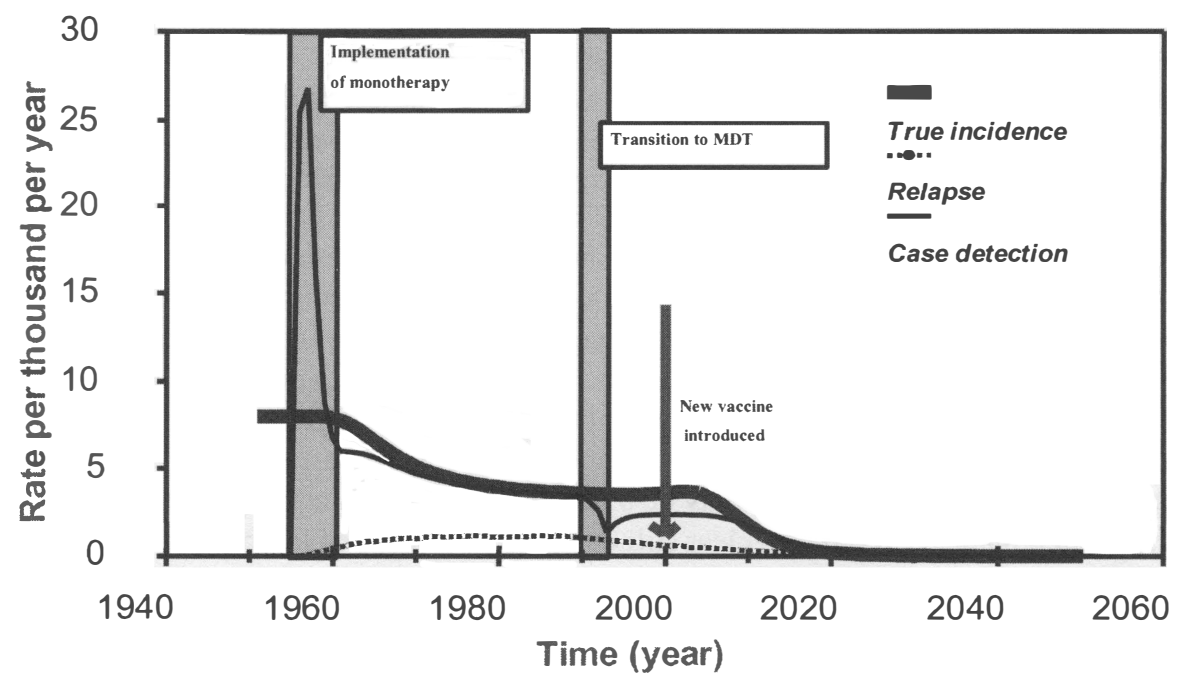

Figure 4. Predicted incidence before and following introduction of an anti-leprosy vaccine that confers $65 \%$ protection. 


\section{What is the meaning of trends in leprosy?}

Observed time-trends for various health conditions are affected by variations of measurement procedures as well as operational factors. Definitions of the conditions may not be constant, as a result of which these projections may not always be very consistent. Thus, the observed trends are affected by both biological and operational factors. The data that are available for calculation of leprosy trends usually come from programmes administered at the national level or, in some situations, from cohort studies and the field areas of some institutions. In any study, initial estimates of leprosy incidence are generally inflated by the backlog of old, previously undetected patients, and are therefore not realistic. The estimates of incidence are also affected by various operational factors, such as whether case finding is active or passive, whether the programme is vertical or integrated, political commitments, and decentralization of the programme. In addition, the allotment of targets for case detection may result in wide fluctuation of case-detection rates.

One also needs to consider the fact that, for the conduct of clinical trials of anti-leprosy drugs, it is now necessary to resort to multicentric studies. Studies of multibacillary leprosy are particularly difficult, because sufficient numbers of cases are no longer available. Hence, one must question whether the observed trends of case detection are real or inflated. The consistently very high new-case detection rates resulting from sample surveys in leprosyendemic regions of India increase the complexity of these observations. It is high time that the confusion is quickly resolved and objective decisions are made, in order to achieve the low levels of leprosy incidence that should lead ultimately to the eradication of leprosy.

\section{References}

1 Gupte MD, Vallishayee RS, Anantharaman DS et al. Comparative leprosy vaccine trial in South India. Indian J Lepr, 1998; 70: 369-388.

2 Gupte MD. Field trials of antileprosy vaccines (Editorial). Ind J Lepr, 1998; 70: 363-367.

3 Tuberculosis Prevention Trial Madras. Trial of BCG vaccines in South India for tuberculosis prevention. Ind J Med Res, 1980; 72: 1-74.

${ }^{4}$ Lwin K, Sundaresan T, Gyi MM et al. BCG vaccination of children against leprosy: fourteen-year findings of the trial in Burma. Bull WHO, 1985; 63: 1069-1078.

5 Convit J, Sampson C, Zuniga M et al. Immunoprophylactic trial with combined Mycobacterium leprae/BCG vaccine against leprosy: Preliminary results. Lancet, 1992; 339: 446-450.

6 Karonga Prevention Trial Group. Randomized controlled trial of single BCG, repeated BCG or combined BCG and killed Mycobacterium le prae vaccine for prevention of leprosy and tuberculosis in Malawi. Lancet, 1996; 348: 17-24.

${ }^{7}$ Fine PEM. South Indian leprosy vaccine trial: Important lessons for mycobacterial immunology. Lepr Rev, 1999; 70: $247-249$.

${ }^{8}$ World Health Organization. Weekly Epdemiol Rec, 1999; 74: 313-320.

9 Gupte MD. Elimination of leprosy: forecasts and projections. Ind J Lepr, 1994; 66: 19-38.

${ }^{10}$ Meima A, Gupte MD, van Oortmarssen GJ, Habbema JDF. Trends in leprosy case detection rates. Int J Lepr, 1997; 65: 305-319.

11 Meima A, Gupte MD, Van Oortmarssen GJ, Habbema JDF. SIMLEP: a simulation model for leprosy transmission and control. Int J Lepr, 1999; 67: 215-230.

12 Gupte MD, Kishore KB, Elangovan A, Arockiasamy J. Modelling epidemiology of leprosy. Ind J Lepr, February 2000 (accepted for publication).

\section{DISCUSSION}

Dr Colston: I wish to ask a practical question. In your recent trial, you found that the 
protective efficacy of one was $65 \%$. We've heard Dr Noordeen say that it has become impossible to carry out large vaccine trials in leprosy. Could one carry out small trials to examine whether or not the vaccine would be capable of reducing the incidence of the disease?

Dr Gupte: I don't recommend phase-III studies of the vaccine. On the other hand, several unanswered questions remain. For example, the immune response to the vaccine has not been investigated at all. Studies of these aspects of the vaccine could be examined on a small scale.

A field study of the vaccine does not appear indicated. However, the question of feasibility remains open. To examine this question requires a phase IV study, which must be much larger than a phase III study, so large, in fact, that it will need to be carried out simultaneously in several parts of India. Such a study will be possible, because it need not be as rigorous as a phase III study — a study to measure efficacy; it could be carried out under the conditions of a leprosy-control programme.

Dr Noordeen: I believe that Dr Gupte has raised an important issue-that of eradication. Personally, I should like to see leprosy eradicated. But as a public health manager, I must ask the question: 'Is it necessary to eradicate leprosy?' Eradication of poliomyelitis or smallpox has great significance; if a few cases of either of these diseases remain, the disease can quickly become epidemic again. On the other hand, a quick resurgence of leprosy appears unlikely indeed. It will become more and more difficult to convince those responsible for allocation of resources that eradication of leprosy should be a priority.

Dr Gupte: I agree. It will become increasingly difficult for governments to allocate the necessary resources as the leprosy caseload becomes smaller and smaller, particularly as integration of leprosy into the general health services is occurring everywhere.

Professor Smith: If we in the research community are not to commit ourselves to the search for the tools necessary to achieve the eradication of leprosy, then, based on the predictions from your simulation model, we must inform the various funding agencies that they must remain committed to additional decades of programmes of detection and MDT.

Professor Britton: In this portion of the Workshop, we are discussing trends of leprosy. Would you comment upon the other epidemiological markers employed in leprosy, such as the incidence among children, the rate of disability, and the ratio of MB to PB cases? You presented a rather contrary view of what is occurring in India, when you called attention to the continuing high new-case detection rate in Tamil Nadu, whereas, in other parts of India, changes of some of these other markers have occurred. Are these other markers useful indicators of what is happening with regard to transmission?

Dr Gupte: In highly endemic states, such as Tamil Nadu, various case-detection activities have revealed an increasing frequency of single-lesion cases. During the last several years, these cases have represented about $30 \%$ of the new cases detected. Similarly, the proportion of children amounts to $30-40 \%$ of the new cases. However, because the nature of the casedetection activities varies from state to state, with school health surveys conducted in some states but not in others, it is difficult to compare the state-by-state data. The rate of disability among new cases is rather small throughout India-no greater than $5 \%$.

Professor Britton: The taxonomy of the ICRC bacillus is very interesting. Is its genome being studied?

Dr Gupte: The answer to your question is not so simple. The genome of the ICRC bacillus is currently being studied by Dr Katoch at JALMA. The ICMR is also planning to send the organisms to a laboratory outside India for phenotypic and genotypic chacterization.

Dr Klatser: How did you define leprosy in your trial. And in particular, how did you define 'progressive and serious leprosy'? 
Dr Gupte: The progressive and serious forms of leprosy included lepromatous leprosy, BL disease, smear positivity and grade 2 disability. There were only a few of these cases among the newly detected patients. Most of these cases were patients with PB disease and patients with single lesions. The diagnosis of leprosy was based only on clinical criteria.

Professor Grosset: There is no way to diagnose leprosy other than clinical.

Dr Gupte: During the recruitment for this trial, emphasis was placed on sensitivity of diagnosis. Every effort was made to exclude leprosy patients from the trial. On the other hand, during the resurveys, emphasis was placed on specificity; I can say with confidence that all of the newly detected cases were patients with leprosy. 